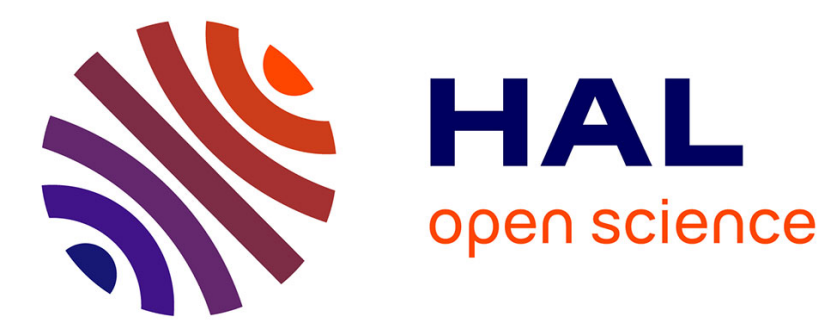

\title{
Event-triggered PI control for continuous plants with input saturation
}

\author{
Luciano G Moreira, L. B Groff, João Manoel Gomes da Silva Jr, Sophie
}

Tarbouriech

\section{> To cite this version:}

Luciano G Moreira, L. B Groff, João Manoel Gomes da Silva Jr, Sophie Tarbouriech. Event-triggered PI control for continuous plants with input saturation. American Control Conference (ACC 2016), Jul 2016, Boston, United States. pp.4251 - 4256, 10.1109/ACC.2016.7525590 . hal-01856308

\section{HAL Id: hal-01856308 https://hal.laas.fr/hal-01856308}

Submitted on 10 Aug 2018

HAL is a multi-disciplinary open access archive for the deposit and dissemination of scientific research documents, whether they are published or not. The documents may come from teaching and research institutions in France or abroad, or from public or private research centers.
L'archive ouverte pluridisciplinaire HAL, est destinée au dépôt et à la diffusion de documents scientifiques de niveau recherche, publiés ou non, émanant des établissements d'enseignement et de recherche français ou étrangers, des laboratoires publics ou privés. 


\title{
Event-triggered PI control for continuous plants with input saturation
}

\author{
L. G. Moreira, L. B. Groff, J. M. Gomes da Silva Jr., S. Tarbouriech
}

\begin{abstract}
This paper proposes a methodology to design stabilizing event-trigger strategies for PI controlled linear continuous-time plants subject to input saturation. Using Lyapunov theory techniques, LMI-based conditions are derived to guarantee regional (or global, when possible) asymptotic stability of the origin. These conditions can be cast in an optimization problem to choose the parameters of the trigger function aiming at reducing the sampling activity, while ensuring the regional stability of the origin with respect to a given set of admissible initial states. Simulation results illustrate the application and potentialities of the method.
\end{abstract}

\section{INTRODUCTION}

Event-triggered control techniques consist in sampling and transmitting data only when a trigger condition occurs [1]. A challenge in this case is to devise trigger conditions that ensure the stability of the closed-loop system under the aperiodic sampling strategy. With this respect, [2] is a widely cited work, which shows that there exist lower bounds for the inter-sampling time of a stabilizing event-triggered control when a threshold on the relative state measurement error is used as trigger condition. Moreover, it is of major interest in networked control systems to develop systematic methods to "tune" the parameters of the trigger condition aiming at reducing the number of samples to deal with the problems of limited bandwidth and energy consumption (mainly in wireless networks). More recently, other issues have also been addressed in the literature. For instance, the co-design between the trigger function and controller gains [3] and the application to nonlinear systems along with the assumption that there is one sensor and one event-trigger generator for each state variable [4].

On the other hand, PI controllers are of special interest due to its massive use in the process industry and applications where constant references and disturbances have to be tracked or rejected. Among the works dealing with eventtriggered PI or PID controlled systems, we can cite [5] and [6] that propose triggering data transmissions when the difference between the current plant output value and the last sampled one crosses a given threshold. Although simulations that give insights on the applicability of this strategy scheme

\footnotetext{
*This work was supported by FAPERGS (MSc scholarship), CAPES (grants STIC-AmSud and PVE), CNPQ (grants PQ 306210/2009-6, UNIV. 480638/2012-8), Brazil, and by ADNEC project STIC-AmSud grant number 13STIC-03

L. G. Moreira, L. B. Groff and J. M. Gomes da Silva Jr. are with Department of Automation and Energy (DELAE) and Pos-Graduation Program in Electrical Engineering (PPGEE), UFRGS, av. Osvaldo Aranha, 103, 90035-190 Porto AlegreRS, Brazil. e-mails: luciano.moreira@ufrgs.br, leonardo.groffeufrgs.br, jmgomesdece.ufrgs.br S. Tarbouriech is with LAAS-CNRS, Université de Toulouse, CNRS, Toulouse, France. e-mail: tarbour@laas.fr
}

are presented, those papers do not give explicit guidance on how to choose the threshold values neither prove the stability of the resulting closed-loop systems. In [7], the authors present a design method for event-triggered PI controllers and LTI plants. Based on the Lyapunov Theory, formal proofs of stability of the closed-loop system under the proposed sampling strategy are provided. The proposed method allows to design all the parameters of the controller and an eventtrigger condition to attain a linear quadratic performance criterion. However, the trigger function used is somewhat complex to calculate, as it uses a matrix of dimension $3(n+1)$. In [8] and [9], stability analysis of event-triggered PI controllers acting on first-order stable plants are presented; the measurement error is used in the trigger criterion without normalizing it with respect to the state. In this case, tracking of constant references with zero steady state error is not achieved.

Furthermore, control input saturation is an ubiquitous phenomenon in real control systems. Thus, it is natural to investigate the use of event-triggered techniques with plants subject to control input saturation. In this case, the closed-loop system becomes nonlinear and for exponentially unstable open-loop systems only regional stabilization is possible to achieve [10]. In this context, considering statefeedback control laws, in [11] algorithms based on linearquadratic criteria are proposed to design periodic, eventtriggered and self-triggered controllers. Lyapunov and hybrid system techniques are used along with generalized sector conditions to ensure exponential stability of the origin and to provide estimates of the region of attraction. Considering a discrete-time framework, saturating state feedback control laws are also addressed in [12]. Regarding PI controllers we can cite [13], which proposes to design an event-triggered controller without taking the saturation into account and then investigates how the saturation impacts the performance of the closed-loop system. The authors show, by means of examples, that the use of anti-windup techniques can reduce the negative effects of saturation. Since the saturation is not taken into account in the design of the trigger function, there is no a priori guarantee that the designed system will still be stable when the saturation effectively occurs. Moreover, no systematic procedure to determine suitable values for the trigger function are presented. In [14] and [15], stability issues under control saturation are addressed considering PI and generic dynamic output feedback controllers. Although the proposed conditions ensure that the trajectories are bounded in an ellipsoidal set, the convergence to the origin (i.e. asymptotic stability) is not guaranteed. It should be pointed out that the problem setup in that paper assumes 
that the output of the controller is continuously applied to the plant (i.e. no sampling and zero order hold are considered), which presupposes that controller and plant are in the same node in a networked implementation.

The present paper addresses the design of an event-trigger strategy for PI controlled continuous-time linear plants subject to input saturation. Differently from [14] and [15], we suppose that plant and controller are in different nodes and that the value of the control signal is kept constant between two sampling instants (i. e. between two successive events). The considered trigger function takes into account a weighted (through the use of generic positive definite matrices) relative distance between the last sampled state and the current continuous one. Based on Lyapunov theory, LMI conditions to ensure the asymptotic stability of the closedloop system (under the event trigger strategy) for a given set of admissible initial states are proposed. Since the weighting matrices of the trigger function appear explicitly in the LMIs, a convex optimization problem aiming at selecting them in order to reduce the sampling activity (i.e. the number of trigger events) is proposed. A numerical example illustrates the application of the method.

Notation: $\mathbb{R}$ represents the set of real numbers. For $v \in \mathbb{R}$, $\operatorname{sat}(v)$ is the classical symmetric saturation function with limits $\pm u_{0}$, with $u_{0}>0 \in \mathbb{R}$. A deadzone non-linearity is defined as $\phi(v) \triangleq \operatorname{sat}(v)-v$; thereof, we can state that $\operatorname{sat}(v)=v+\phi(v) . A^{\prime}$ denotes the transpose of matrix $A$. $\operatorname{He}\{A\} \triangleq A+A^{\prime}$. The symbol * stands for symmetric blocks within a matrix. $\operatorname{tr}(A)$ denotes the trace of matrix $A \cdot \operatorname{diag}(X, Y)$ denotes the block-diagonal matrix composed by the blocks $X$ and $Y . \mathcal{E}(P)=\left\{\xi \in \mathbb{R}^{q} ; \xi^{\prime} P \xi \leq 1\right\}$ denotes an ellipsoid defined from a matrix $P=P^{\prime}>0 \in \mathbb{R}^{q \times q}$.

\section{Problem Statement}

Consider a continuous-time single-input single-output linear plant defined by the following equations:

$$
\left\{\begin{aligned}
\dot{x}_{p}(t) & =A_{p} x_{p}(t)+B_{p} u(t) \\
y(t) & =C_{p} x_{p}(t)
\end{aligned}\right.
$$

where $x_{p}(t) \in \mathbb{R}^{n}$ is the state vector; $u(t) \in \mathbb{R}$ is the input, limited in amplitude such that $-u_{0} \leq u(t) \leq u_{0}$, with $u_{0}>0 ; y(t) \in \mathbb{R}$ is the controlled output; $A_{p}, B_{p}$ and $C_{p}$ are real-valued constant matrices of appropriate dimensions. We assume that the plant is observable and controllable.

The controller is a continuous-time PI controller, defined by the following state space representation:

$$
\left\{\begin{aligned}
\dot{x}_{c}(t) & =-y(t) \\
\nu(t) & =k_{i} x_{c}(t)-k_{p} y(t)
\end{aligned}\right.
$$

where $x_{c}(t) \in \mathbb{R}$ is the controller state; $\nu(t) \in \mathbb{R}$ is the controller output and $k_{p}, k_{i} \in \mathbb{R}$ are the proportional and integral gains, respectively.

We consider a networked control implementation where plant and controller are in separate nodes and are connected through a general purpose network forming the closed-loop system depicted in Figure 1.

At instants determined by an event-trigger generator, a sample of the plant output is sent to the controller node.

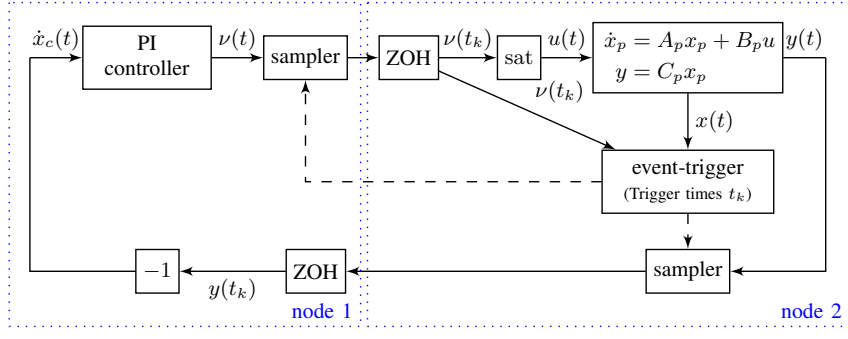

Fig. 1. Closed-loop system diagram

At the same time, the current value of $\nu(t)$ is sent to the plant input and to the event generator. Between two trigger instants, the controller and plant inputs are held at the constant sampled values by means of zero-order holders.

Therefore, the closed-loop system can be represented as follows:

$$
\left\{\begin{aligned}
\dot{x}_{p}(t) & =A_{p} x_{p}(t)+B_{p} u(t) & & \\
y(t) & =C_{p} x_{p}(t) & & \\
\dot{x}_{c}(t) & =-y\left(t_{k}\right) & & \forall t \in\left[t_{k}, t_{k+1}\right) \\
\nu(t) & =k_{i} x_{c}(t)-k_{p} y\left(t_{k}\right) & & \forall t \in\left[t_{k}, t_{k+1}\right) \\
u(t) & =\operatorname{sat}\left(\nu\left(t_{k}\right)\right) & & \forall t \in\left[t_{k}, t_{k+1}\right)
\end{aligned}\right.
$$

where $t_{k}, k=0,1,2,3, \ldots$ are the triggering times. We consider $t_{0}=0$. Note that the equilibrium point is assumed to be the origin. If the system is subject to a non-zero constant reference, the equilibrium is not the origin anymore, but a variable translation can be done and the stability analysis will be the same (see details in [7]).

Due to the control input saturation, the overall behavior of the closed-loop system is nonlinear. In this case, defining the state vector $x=\left[\begin{array}{ll}x_{p}^{\prime} & x_{c}^{\prime}\end{array}\right]^{\prime}$, the region of attraction for (3) can be defined as follows [16]:

Definition 1: The region of attraction (RA) of the origin for the system (3) is the set of all initial states $x_{0} \in \mathbb{R}^{(n+1)}$ for which $x(0)=x_{0} \Longrightarrow x(t) \rightarrow 0$ as $t \rightarrow \infty$.

In words, the RA is the set of all initial conditions whose trajectories converge to the origin. Nevertheless the exact characterization of the RA is, in general, a complex task ([16]-[18]). Thus, it is useful to characterize subsets of the RA that have an analytical representation, such as ellipsoidal and polyhedral sets. These subsets can be used as estimates of the RA and are called regions of asymptotic stability (RAS) [16].

Considering system (3), we aim at designing an eventtriggered control strategy (i. e. that defines the trigger instants $t_{k}$ ) to reduce the number of messages exchanged between the nodes, so that we can save energy and communication bandwidth, while keeping the closed-loop system stable. Hence we can formally state the problem we want to address as follows:

Problem 1: Devise an event-triggered strategy for the closed-loop system (3) guaranteeing the regional asymptotic stability of the origin for initial conditions in a given subset $\mathcal{X}_{0}$ of the RA of the closed-loop system, while reducing the number of data transmission events between the sensor/plant node and the controller node. 


\section{Continuous-Time SyStem STABILITY}

Before analyzing the event-triggered control strategy, we recall some stability conditions for the continuous-time system composed by the direct connection between (1) and (2), through a saturation function, i.e. $u(t)=\operatorname{sat}(\nu(t))$, which leads to the following system:

$$
\left\{\begin{array}{l}
\dot{x}_{p}(t)=A_{p} x_{p}(t)+B_{p} \operatorname{sat}\left(k_{i} x_{c}(t)-k_{p} C_{p} x_{p}(t)\right) \\
\dot{x}_{c}(t)=-C_{p} x_{p}(t)
\end{array}\right.
$$

We assume that the gains $k_{i}$ and $k_{p}$ have been designed such that (4) is regionally stable in a set $\mathcal{X}_{0}$. This is guaranteed if $k_{i}$ and $k_{p}$ are such that there exist a scalar $\varsigma>0$, matrices $\bar{W}=\bar{W}^{\prime}>0 \in \mathbb{R}^{(n+1) \times(n+1)}, \bar{Z} \in \mathbb{R}^{1 \times(n+1)}$ satisfying the following linear matrix inequalities (see [16] for details):

$$
\begin{array}{r}
{\left[\begin{array}{cc}
\operatorname{He}\{(A+B K) \bar{W}\} & \varsigma B-\bar{Z}^{\prime} \\
* & -2 \varsigma
\end{array}\right]<0} \\
{\left[\begin{array}{cc}
\bar{W} & \bar{W} K^{\prime}-\bar{Z} \\
* & u_{0}^{2}
\end{array}\right] \geq 0}
\end{array}
$$

and $\mathcal{X}_{0} \subset \mathcal{E}\left(\bar{W}^{-1}\right)$, with

$$
A \triangleq\left[\begin{array}{cc}
A_{p} & 0 \\
-C_{p} & 0
\end{array}\right] ; B \triangleq\left[\begin{array}{c}
B_{p} \\
0
\end{array}\right] ; K \triangleq\left[\begin{array}{ll}
-k_{p} C_{p} & k_{i}
\end{array}\right]
$$

In this case, it follows that $V(x)=x^{\prime} P x$, with $P=\bar{W}^{-1}$ is such that $\dot{V}(x)<0, \forall x \in \mathcal{E}(P)$, i. e. $\mathcal{E}(P)$ is a contractive domain for the closed-loop system (4).

\section{EVENT-TRIGGER STRATEGY}

In this section, we propose an event-trigger strategy and provide stability conditions by means of a quadratic Lyapunov function.

Defining $\delta(t) \triangleq x\left(t_{k}\right)-x(t)$, with $x(t)$ being the state of the closed-loop system (in our case, the plant and controller system combined), it is shown in [2] that one can use the triggering criterion presented below to ensure that an eventtriggered control system like (3) is stable:

$$
\begin{aligned}
& \text { if }\|\delta(t)\|=\sigma_{0}\|x(t)\| \text { then } \\
& \quad \text { trigger } \\
& \text { end if }
\end{aligned}
$$

where $\|\cdot\|$ denotes the Euclidean norm and $\sigma_{0}$ is a scalar selected from an interval of values determined by the dynamics of the system.

In this paper, we extend this idea by using the following generalized criterion:

$$
\begin{aligned}
& \text { if } \delta^{\prime}(t) Q_{\delta} \delta(t)>x^{\prime}(t) Q_{x} x(t) \text { then } \\
& \quad \text { trigger } \\
& \text { end if }
\end{aligned}
$$

where $Q_{\delta}$ and $Q_{x}$ are symmetric positive definite matrices of dimension $(n+1) \times(n+1)$. With this generalization, we add degrees of freedom and we expect to allow a larger reduction in the sampling activity. Notice that we can rewrite our event-trigger strategy as follows:

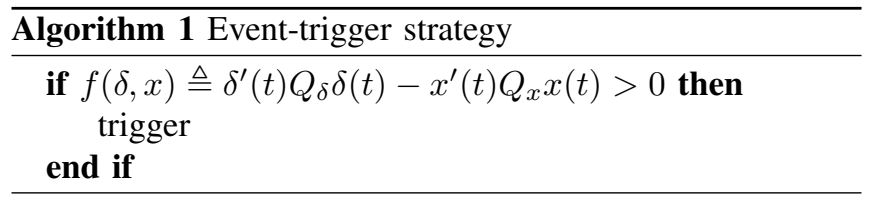

The term $\delta^{\prime}(t) Q_{\delta} \delta(t)-x^{\prime}(t) Q_{x} x(t)$ is a relative measure of the deviation between the last sampled state and the current state; $Q_{\delta}$ and $Q_{x}$ act as weights on this measure. The relation between these matrices plays a role similar to the one of $\sigma_{0}$ in [2], in the sense that the "larger" $Q_{x}$ and the "smaller" $Q_{\delta}$ are, the more we let the current state deviate from the last sampled one and the less sampling activity is expected. To illustrate this, notice that if we choose $Q_{x}=\sigma I$ and $Q_{\delta}=\mu I$ we basically retrieve the criterion in [2] with $\sigma_{0}=\sigma / \mu$ and, as shown in that paper, the larger $\sigma_{0}$ the less sampling activity is expected.

Let $A, B$ and $K$ be given matrices defined as in (6), with $K$ verifying (5), and define

$$
A_{\delta} \triangleq\left[\begin{array}{cc}
0 & 0 \\
-C_{p} & 0
\end{array}\right]
$$

Now we derive conditions that ensure the asymptotic stability of the origin when the event-trigger strategy described in Algorithm 1 is used.

Theorem 1: If there exist a scalar $\varsigma>0$, matrices $W=$ $W^{\prime}>0 \in \mathbb{R}^{(n+1) \times(n+1)}, \bar{Q}_{x}=\bar{Q}_{x}^{\prime}>0 \in \mathbb{R}^{(n+1) \times(n+1)}$, $Q_{\delta}=Q_{\delta}^{\prime}>0 \in \mathbb{R}^{(n+1) \times(n+1)}$ and $Z, G_{2} \in \mathbb{R}^{1 \times(n+1)}$ such that the following LMIs are satisfied:

$$
\begin{gathered}
{\left[\begin{array}{cccc}
\mathrm{He}\{(A+B K) W\} & W & \left(A_{\delta}+B K\right) & \varsigma B-Z^{\prime} \\
* & -\bar{Q}_{x} & 0 & 0 \\
* & * & -Q_{\delta} & -G_{2}^{\prime} \\
* & * & * & -2 \varsigma
\end{array}\right]<0} \\
\\
{\left[\begin{array}{ccccc}
W & 0 & W K^{\prime}-Z^{\prime} & W \\
* & Q_{\delta} & K^{\prime}-G_{2}^{\prime} & 0 \\
* & * & u_{0}^{2} & 0 \\
* & * & * & \bar{Q}_{x}
\end{array}\right]>0}
\end{gathered}
$$

then, $\mathcal{E}\left(W^{-1}\right)=\left\{x \in \mathbb{R}^{n+1} ; x^{\prime} W^{-1} x \leq 1\right\}$ is a RAS for the system (3) under the sampling strategy given by Algorithm 1, with $Q_{x}=\bar{Q}_{x}^{-1}$.

Proof: Considering $\delta_{p}(t) \triangleq x_{p}\left(t_{k}\right)-x_{p}(t), \delta_{c}(t) \triangleq$ $x_{c}\left(t_{k}\right)-x_{c}(t)$ and $\operatorname{sat}(\nu)=\nu+\phi(\nu)$, note that between two trigger events, that is for $t \in\left(t_{k}, t_{k+1}\right)$, we can re-write (3) as follows:

$$
\left\{\begin{aligned}
\dot{x}_{p}(t)= & A_{p} x_{p}(t)+B_{p} u(t) \\
\dot{x}_{c}(t)=- & C_{p} x_{p}(t)-C_{p} \delta_{p}(t) \\
u(t)= & -k_{p} C_{p}\left[x_{p}(t)+\delta_{p}(t)\right]+k_{i}\left[x_{c}(t)+\delta_{c}(t)\right] \\
& \quad+\phi\left(\nu\left(t_{k}\right)\right) \\
y(t)= & C_{p} x_{p}(t)
\end{aligned}\right.
$$

Using now $A, B$ and $K$ as defined in (6) and considering $x \triangleq\left[\begin{array}{l}x_{p} \\ x_{c}\end{array}\right], \delta \triangleq\left[\begin{array}{l}\delta_{p} \\ \delta_{c}\end{array}\right]$ and $C \triangleq\left[\begin{array}{ll}C_{p} & 0\end{array}\right]$, we can rewrite (9) as follows, where we dropped the time dependence to simplify the notation:

$$
\left\{\begin{array}{l}
\dot{x}=(A+B K) x+\left(A_{\delta}+B K\right) \delta+B \phi(K(x+\delta)) \\
y=C x
\end{array}\right.
$$


Considering a quadratic Lyapunov function candidate $V(x(t))=x^{\prime}(t) P x(t)$, it follows that

$$
\begin{aligned}
\dot{V}(x)= & x^{\prime} \operatorname{He}\{P(A+B K)\} x \\
& +2 x^{\prime} P\left(A_{\delta}+B K\right) \delta+2 x^{\prime} P B \phi
\end{aligned}
$$

Notice that the argument of $\phi$, i.e. $\nu\left(t_{k}\right)$, can be written as

$$
K(x+\delta)=\left[\begin{array}{ll}
K & K
\end{array}\right]\left[\begin{array}{l}
x \\
\delta
\end{array}\right] \triangleq K_{a} x_{a}
$$

where $K_{a} \triangleq\left[\begin{array}{ll}K & K\end{array}\right]$ and $x_{a} \triangleq\left[\begin{array}{l}x \\ \delta\end{array}\right]$.

Hence, provided that $x_{a}$ belongs to the region $\mathcal{S}_{0}=\left\{x_{a} \in\right.$ $\left.\mathbb{R}^{2(n+1)} ;\left|K_{a} x_{a}-G_{a} x_{a}\right| \leq u_{0}\right\}$, the following generalized sector condition ([16], [19]) is verified with respect to the deadzone nonlinearity $\phi$ :

$$
\tau \phi^{\prime}\left(K_{a} x_{a}\right)\left(\phi\left(K_{a} x_{a}\right)+G_{a} x_{a}\right) \leq 0
$$

with $\tau$ being a positive scalar and $G_{a}=\left[\begin{array}{ll}G_{1} & G_{2}\end{array}\right]$ a free matrix of appropriate dimensions.

Assuming $x_{a} \in \mathcal{S}_{0}$ and applying (13), we can write

$$
\dot{V}(x) \leq \dot{V}(x)-2 \tau \phi^{\prime}\left(K_{a} x_{a}\right)\left(\phi\left(K_{a} x_{a}\right)+G_{a} x_{a}\right)
$$

Thus, from (11) and (12), (14) can be written as follows:

$$
\dot{V}(x) \leq\left[\begin{array}{lll}
x^{\prime} & \delta^{\prime} & \phi^{\prime}
\end{array}\right] M\left[\begin{array}{lll}
x^{\prime} & \delta^{\prime} & \phi^{\prime}
\end{array}\right]^{\prime}
$$

with

$$
M=\left[\begin{array}{ccc}
\operatorname{He}\{P(A+B K)\} & P\left(A_{\delta}+B K\right) & P B-\tau G_{1}^{\prime} \\
* & 0 & -\tau G_{2}^{\prime} \\
* & * & -2 \tau
\end{array}\right]
$$

From Algorithm 1, it follows that $\delta^{\prime} Q_{\delta} \delta-x^{\prime} Q_{x} x \leq 0$. Then, if the following matrix inequality is verified:

$$
\left[\begin{array}{ccc}
\operatorname{He}\{P(A+B K)\}+Q_{x} & P\left(A_{\delta}+B K\right) & P B-\tau G_{1}^{\prime} \\
* & -Q_{\delta} & -\tau G_{2}^{\prime} \\
* & * & -2 \tau
\end{array}\right]<0
$$

from (15), we can conclude that $\dot{V}(x)<\delta^{\prime} Q_{\delta} \delta-x^{\prime} Q_{x} x \leq 0$ for $t \in\left(t_{k}, t_{k+1}\right)$, provided $x_{a} \in \mathcal{S}_{0}$. Now pre- and postmultiplying (16) by $\operatorname{diag}(W, I, \varsigma)$ with $W=P^{-1}, \varsigma=$ $\tau^{-1}$, considering the variable changes $Z=G_{1} W$ and then applying Schur's complement, we retrieve matrix inequality (7), with $\bar{Q}_{x}=Q_{x}^{-1}$. Thus, the satisfaction of (7) guarantees $\dot{V}(x)<0$ for $t \in\left(t_{k}, t_{k+1}\right)$, provided $x_{a} \in \mathcal{S}_{0}$.

At the instants $t=t_{k}, \delta(t)$ is zero and the system reduces to the continuous-time (4). In this case, it can be verified that (16) implies (5) and it follows that $\dot{V}\left(x\left(t_{k}\right)\right)<0$, provided $x_{a}\left(t_{k}\right) \in \mathcal{S}_{0}$.

Now we show that (8) guarantees that $x_{a} \in \mathcal{S}_{0}, \forall t \geq$ 0 , provided $x(0) \in \mathcal{E}(P)$. Pre- and post-multiplying (8) by $\operatorname{diag}(P, I, 1, I)$, applying Schur's complement twice and recalling that $Z=G_{1} W$, the following relation is verified

$$
\left[\begin{array}{cc}
P-Q_{x} & 0 \\
0 & Q_{\delta}
\end{array}\right]-\left[K-G_{1} K-G_{2}\right]^{\prime} \frac{1}{u_{0}^{2}}\left[K-G_{1} K-G_{2}\right]>0
$$

Pre- and post-multiplying (17) by $\left[\begin{array}{ll}x^{\prime} & \delta^{\prime}\end{array}\right]$ and $\left[\begin{array}{l}x \\ \delta\end{array}\right]$ respectively, (17) implies that

$$
\begin{aligned}
& x^{\prime} P x+\delta^{\prime} Q_{\delta} \delta-x^{\prime} Q_{x} x \\
& -x_{a}^{\prime}\left[K-G_{1} K-G_{2}\right]^{\prime} \frac{1}{u_{0}^{2}}\left[K-G_{1} K-G_{2}\right] x_{a}>0
\end{aligned}
$$

Hence, if $t \in\left(t_{k}, t_{k+1}\right)$, since $\delta^{\prime} Q_{\delta} \delta-x^{\prime} Q_{x} x \leq 0$, we conclude that $x_{a}(t) \in S_{0}$ if $x(t) \in \mathcal{E}(P)$. On the other hand, at $t=t_{k}$, we have $\delta(t)=0$ and it also follows that $x_{a}\left(t_{k}\right) \in \mathcal{S}_{0}$ if $x\left(t_{k}\right) \in \mathcal{E}(P)$. Thus, we can conclude that if $x(0) \in \mathcal{E}(P)$, (7) along with (8) effectively ensures that $x_{a}(t) \in \mathcal{S}_{0}$ and $\dot{V}(x)<0, \forall t \geq 0$, which ensures that $\mathcal{E}(P)$ is an invariant and contractive set with respect to the system (3), being included in its region of attraction.

Theorem 1 ensures the regional asymptotic stability of the closed-loop system. Actually, it can be applied to both stable or unstable plants. Nevertheless, if $A_{p}$ is Hurwitz, global stability can be achievable [10]. A sufficient condition for the global stability of the closed-loop system under the event-trigger strategy can therefore be stated as a corollary to Theorem 1 as follows.

Corollary 1: If there exist a scalar $\varsigma>0$, matrices $W=$ $W^{\prime}>0 \in \mathbb{R}^{(n+1) \times(n+1)}, \bar{Q}_{x}=\bar{Q}_{x}^{\prime}>0 \in \mathbb{R}^{(n+1) \times(n+1)}$ and $Q_{\delta}=Q_{\delta}^{\prime}>0 \in \mathbb{R}^{(n+1) \times(n+1)}$ such that the following LMI is satisfied:

$$
\left[\begin{array}{cccc}
\operatorname{He}\{(A+B K) W\} & W & \left(A_{\delta}+B K\right) & \varsigma B-W K^{\prime} \\
* & -\bar{Q}_{x} & 0 & 0 \\
* & * & -Q_{\delta} & -K^{\prime} \\
* & * & * & -2 \varsigma
\end{array}\right]<0
$$

then, the origin of system (3) is globally asymptotically stable under the sampling strategy given by Algorithm 1, with $Q_{x}=\bar{Q}_{x}^{-1}$.

Proof: It follows the same lines as the proof of Theorem 1 but applying a global generalized sector condition, i.e., using $G_{a}=K_{a}$, which implies $G_{1}=G_{2}=K$.

Remark 1: It is worth noticing that the possibility of Zeno solutions is eliminated taking into account that the eventtrigger function satisfies:

$$
\|\delta(t)\| \leq \frac{\lambda_{\max }\left(Q_{x}\right)}{\lambda_{\min }\left(Q_{\delta}\right)}\|x(t)\|
$$

Hence, the same arguments used in [2] can be applied here to show that the inter-sampling times are lower bounded.

Remark 2: Notice that the plant and controller states need to be available to implement the event trigger criterion defined by Algorithm 1. Considering that the controller and the event generator run in different nodes, the controller state can be transmitted to the event generator only at the instants $t_{k}$. Then, assuming the controller sends its state at instants $t_{k}, x_{c}(t)$ can be recovered, as in [7], using:

$$
x_{c}(t)=x_{c}\left(t_{k}\right)-\int_{t_{k}}^{t} y\left(t_{k}\right) d t=x_{c}\left(t_{k}\right)-y\left(t_{k}\right)\left(t-t_{k}\right)
$$

In numerous applications only part of the plant state is measured. In this case, since $y(t)$ is assumed to be continuously 
available to the event generator, $x_{p}(t)$ can be recovered through a Luenberger observer:

$$
\hat{x}_{p}(t)=A_{p} \hat{x}_{p}+B_{p} u(t)+L\left(C_{p} \hat{x}_{p}-y(t)\right)
$$

where $\hat{x}_{p}$ is the estimated state.

Remark 3: In [14] and [15] it is assumed that the controller output is continuously applied to the plant. In this scenario, the closed-loop system is given by:

$$
\left\{\begin{aligned}
\dot{x}_{p}(t) & =A_{p} x_{p}(t)+B_{p} u(t) \\
y(t) & =C_{p} x_{p}(t) \\
\dot{x}_{c}(t) & =-y\left(t_{k}\right) \\
u(t) & =\operatorname{sat}(\nu(t)) \\
\nu(t) & =k_{i} x_{c}(t)-k_{p} y\left(t_{k}\right) \quad \forall t \in\left[t_{k}, t_{k+1}\right)
\end{aligned}\right.
$$

Our approach can be easily adapted to cope with this case, i.e. similar conditions to (7) and (8) can be obtained from the same steps used in the proof of Theorem 1. Particularly in this case, the trigger function can be defined only in terms of the plant and controller outputs. However, the possibility of Zeno solutions occurrence has to be carefully studied.

\section{OPTIMIZATION PROBLEMS}

Consider a given region of admissible initial states $\mathcal{X}_{0}$. If $\mathcal{X}_{0} \subset \mathcal{E}(P)$ and conditions of Theorem 1 are satisfied, we conclude that $\mathcal{X}_{0}$ is also included in the region of attraction of the closed-loop system. To solve Problem 1, the idea is also to reduce the number of transmission events, i. e., reduce the number of sampling instants.

As observed in Section IV, in order to reduce the sampling activity, one should aim at finding $Q_{x}$ as "large" as possible and $Q_{\delta}$ as "small" as possible, while ensuring that the closed-loop system under the event-trigger strategy given in Algorithm 1 is stable. Considering that $\mathcal{X}_{0}$ is specified as an ellipsoid $\mathcal{E}\left(P_{0}\right)=\left\{x \in \mathbb{R}^{(n+1)} ; \quad x^{\prime} P_{0} x \leq 1\right\}$, this goal can be achieved from the following convex optimization problem:

$$
\begin{aligned}
& \min \left(\operatorname{tr}\left(Q_{\delta}+\bar{Q}_{x}\right)\right) \\
& \text { subject to: (7), (8), }\left[\begin{array}{cc}
W & I \\
I & P_{0}
\end{array}\right]>0
\end{aligned}
$$

Note that the last constraint in (24) ensures that $\mathcal{X}_{0} \subset$ $\mathcal{E}(P)$. Also note that this optimization problem, although leading to a reduction in the number of events, does not guarantee that the minimum possible is achieved.

\section{NUMERICAL EXAMPLE}

Consider the following unstable plant:

$$
\left\{\begin{array}{l}
\dot{x}(t)=\left[\begin{array}{ll}
0 & 1 \\
4 & 0
\end{array}\right] x(t)+\left[\begin{array}{l}
1 \\
0
\end{array}\right] \operatorname{sat}(u(t)) \\
y(t)=\left[\begin{array}{ll}
1 & 1
\end{array}\right] x(t)
\end{array}\right.
$$

We choose controller gains $k_{p}=18$ and $k_{i}=19$ so that the system without saturation and with a continuoustime controller is stabilized with closed-loop poles in -10.2 , -6.68 and -1.11 .

We consider $\dot{\mathcal{X}}_{0}=\mathcal{E}\left(P_{0}\right)$, with $P_{0}=\operatorname{diag}\left(4,4,10^{6}\right)$. Then we apply the optimization problem (24) proposed in the
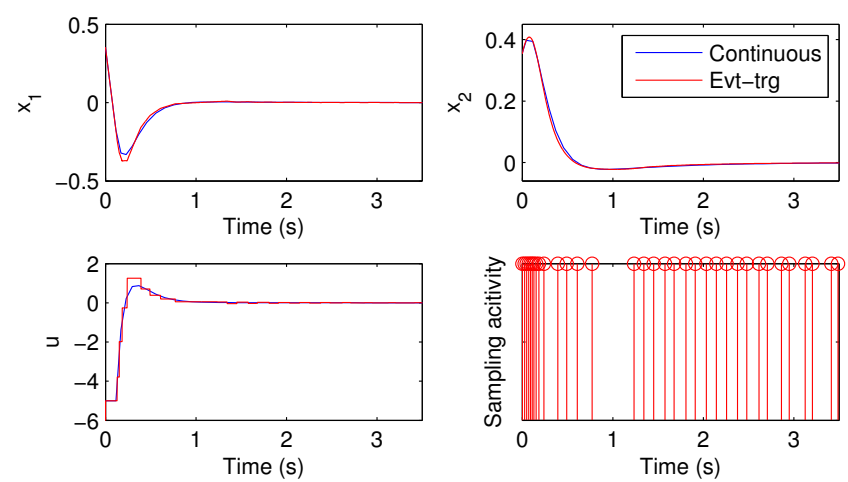

Fig. 2. Simulation results - Comparison between continuous and eventtriggered systems

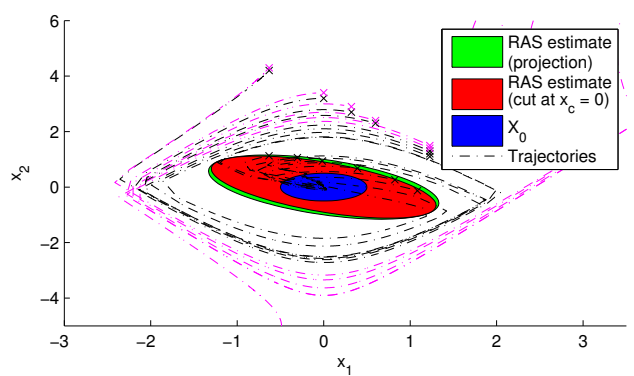

Fig. 3. RAS with event-triggered control

previous section, with an additional constraint $Q_{\delta} \leq 0.01 I$ in order to prevent $Q_{\delta}$ from being ill-conditioned, obtaining:

$Q_{x}=\left[\begin{array}{ccc}0.2360 & -0.1015 & -0.05127 \\ * & 0.4291 & 0.01756 \\ * & * & 1.643\end{array}\right]$
$Q_{\delta}=\left[\begin{array}{ccc}11.33 & 11.32 & -10.72 \\ * & 11.33 & -10.72 \\ * & * & 12.49\end{array}\right] \quad P=\left[\begin{array}{ccc}0.9049 & 0.6176 & -0.8064 \\ * & 1.219 & -0.9665 \\ * & * & 4.240\end{array}\right]$

Figure 2 shows results of a simulation with $x(0)=$ $\left[\begin{array}{lll}\sqrt{2} / 4 & \sqrt{2} / 4 & 0\end{array}\right]^{\prime}$. Only 40 trigger events are needed in the time interval $[0,5]$ and the minimum inter-sampling time is $19 \mathrm{~ms}$. For comparison purposes, the response considering a continuous-time implementation is also depicted in this figure. We can observe that there is no significant degradation on the system performance when compared to the continuous case. As a matter of comparison, if one uses a periodic sampled control law with a sampling period that achieves the same number of samples as the event-triggered, i.e. a sampling period around $100 \mathrm{~ms}$, the trajectory for the same initial condition diverges. Figure 3 shows the projection of the set $\mathcal{E}(P)$ obtained for the event-triggered controller along with the projections of some convergent and some divergent trajectories, in black and in magenta, respectively. Note that $\mathcal{E}(P)$ contains $\mathcal{X}_{0}$, as required.

We consider now a more stringent specification for $\mathcal{X}_{0}$, by choosing $P_{0}=\operatorname{diag}\left(1,1,10^{6}\right)$. Figure 4 shows the projection of the set $\mathcal{E}(P)$ obtained. One can see that $\mathcal{E}(P)$ now is closer to $\mathcal{X}_{0}$. Figure 5 shows results of a simulation with $x_{0}=\left[\begin{array}{lll}\sqrt{2} / 2 & \sqrt{2} / 2 & 0\end{array}\right]^{\prime}$. Now 48 trigger events are needed in 


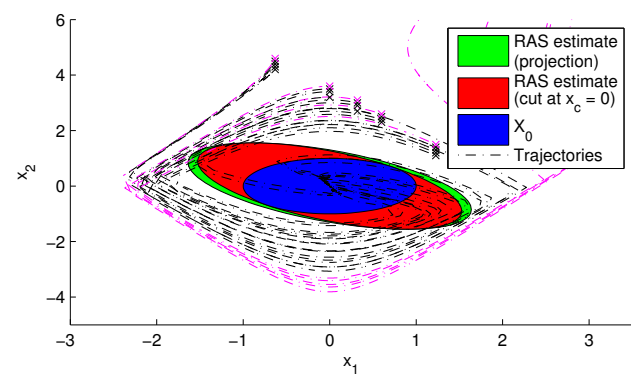

Fig. 4. RAS with event-triggered control - Large $\mathcal{X}_{0}$
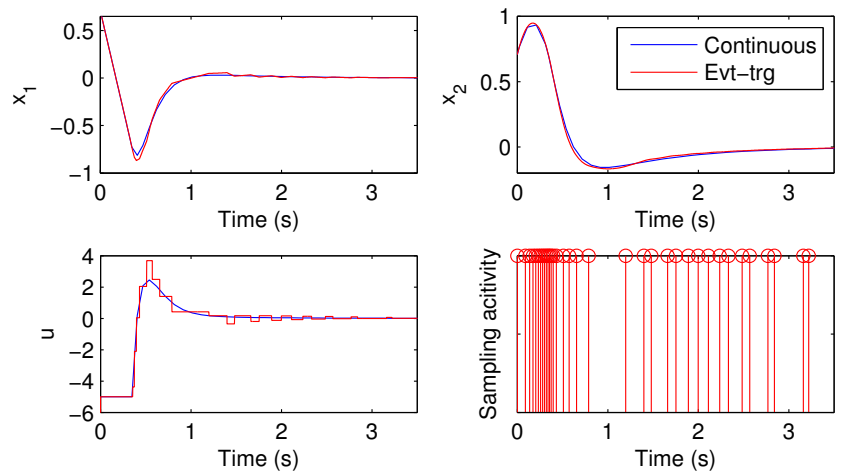

Fig. 5. Simulation results - Comparison between continuous and eventtriggered systems - Large $\mathcal{X}_{0}$

the time interval $[0,5]$ and the minimum inter-sampling time is $17 \mathrm{~ms}$; a slight worse performance than the less restrictive $\mathcal{X}_{0}$ specification. One can also observe a slight degradation on the system performance when compared to the continuous case.

\section{CONCLUDING REMARKS}

In this paper we proposed a systematic methodology to design event-triggered strategies for PI controlled plants subject to input saturation. Differently from previous work, we suppose that plant and controller are in different nodes in a network and that the value of the plant input is kept constant between two trigger instants, i.e. when the trigger condition is verified the plant output is transmitted to the controller and an updated control signal is transmitted to the plant. The method guarantees local asymptotic stability of the origin for a given set of initial conditions and can be easily extended to guarantee global stability for stable plants. Since the derived conditions are in an LMI form, a convex optimization problem is proposed to compute the trigger function parameters aiming at reducing the sampling activity while guaranteeing the stability of the closed-loop system.

The extension of the proposed approach to include antiwindup compensation, the co-design of the trigger function and controller parameters and incorporating the ideas from [20] to handle periodic event-triggered control are subjects of ongoing work.

\section{REFERENCES}

[1] W. Heemels, K. Johansson, and P. Tabuada, "An introduction to eventtriggered and self-triggered control," in 51st IEEE Conference on Decision and Control (CDC), Dec 2012, pp. 3270-3285.

[2] P. Tabuada, "Event-triggered real-time scheduling of stabilizing control tasks," IEEE Transactions on Automatic Control, vol. 52, no. 9, pp. 1680-1685, Sept 2007.

[3] M. Abdelrahim, R. Postoyan, J. Daafouz, and D. Nesic, "Co-design of output feedback laws and event-triggering conditions for linear systems," in 53rd IEEE Conference on Decision and Control (CDC), Dec 2014, pp. 3560-3565.

[4] P. Tallapragada and N. Chopra, "Decentralized event-triggering for control of nonlinear systems," IEEE Transactions on Automatic Control, vol. 59, no. 12, pp. 3312-3324, 2014.

[5] S. Durand and N. Marchand, "Further results on event-based PID controller," in 2009 European Control Conference (ECC), Aug 2009, pp. 1979-1984.

[6] _ , "An Event-Based PID Controller With Low Computational Cost," in 8th International Conference on Sampling Theory and Applications (SampTA'09), L. Fesquet and B. Torrésani, Eds., Marseille, France, May 2009, p. Special session on Sampling and Industrial Applications.

[7] J. M. Gomes Da Silva Jr., W. F. Lages, and D. G. Sbarbaro, "Eventtriggered PI control design," in 19th IFAC World Congress (IFAC WC 2014), Cape Town, South Africa, 2014, pp. 6947-6952.

[8] U. Tiberi, J. Araujo, and K. H. Johansson, "On event-based PI control of first-order processes," in 2nd IFAC Conference on Advances in PID Control, PID'12, Brescia, Italy, Mar 2012, pp. 448-453.

[9] M. Beschi, S. Dormido, J. Sánchez, and A. Visioli, "Event-based PI controller with exponential thresholds," in 19th IFAC World Congress (IFAC WC 2014), Cape Town, South Africa, Aug. 2014, pp. 57665771

[10] H. Sussmann, E. Sontag, and Y. Yang, "A general result on the stabilization of linear systems using bounded controls," IEEE Transactions on Automatic Control, vol. 39, no. 12, pp. 2411-2425, Dec 1994.

[11] A. Seuret, C. Prieur, S. Tarbouriech, and L. Zaccarian, "Eventtriggered control with LQ optimality guarantees for saturated linear systems," in 9th IFAC Symposium on Nonlinear Control Systems (NOLCOS 2013), Toulouse, France, Sep. 2013, p. 6p.

[12] W. Wu, S. Reimann, and S. Liu, "Event-triggered control for linear systems subject to actuator saturation," in 19th IFAC World Congress (IFAC WC 2014), Cape Town, South Africa, Aug. 2014.

[13] D. Lehmann and K. H. Johansson, "Event-triggered PI control subject to actuator saturation," in 2nd IFAC Conference on Advances in PID Control, PID'12, Brescia, Italy, pp. 430-435.

[14] D. Lehmann, G. Kiener, and K. Johansson, "Event-triggered PI control: Saturating actuators and anti-windup compensation," in 51st IEEE Conference on Decision and Control (CDC), Dec 2012, pp. 65666571

[15] G. Kiener, D. Lehmann, and K. Johansson, "Actuator saturation and anti-windup compensation in event-triggered control," Discrete Event Dynamic Systems, vol. 24, no. 2, pp. 173-197, 2014

[16] S. Tarbouriech, G. Garcia, J. M. Gomes Da Silva Jr., and I. Queinnec, Stability and Stabilization of Linear Systems with Saturating Actuators. Springer, 2011.

[17] H. K. Khalil, Nonlinear systems. Upper Saddle River, (N.J.): Prentice Hall, 1996

[18] R. Genesio, M. Tartaglia, and A. Vicino, "On the estimation of asymptotic stability regions: State of the art and new proposals," IEEE Transactions on Automatic Control, vol. 30, no. 8, pp. 747-755, 1985.

[19] J. M. Gomes Da Silva Jr. and S. Tarbouriech, "Antiwindup design with guaranteed regions of stability: an LMI-based approach," IEEE Transactions on Automatic Control, vol. 50, no. 1, pp. 106-111, Jan 2005.

[20] E. Aranda-Escolastico, M. Guinaldo, and S. Dormido, "A novel approach for periodic event-triggering based on general quadratic functions," in 2015 International Conference on Event-based Control, Communication, and Signal Processing (EBCCSP), Krakow, Poland, June 2015, pp. 1-6. 